Acta Theriologica, Suppl. 5: 53-62, 1998.

PL ISSN 0001-7051

\title{
Genetic markers for the detection of quantitative trait loci with special consideration of body weight and fat
}

\author{
Gudrun A. BROCKMANN*, Ulla RENNE, \\ Kathrin KOPPLOW and Paramananda DAS
}

\begin{abstract}
Brockmann G. A., Renne U., Kopplow K. and Das P. 1998. Genetic markers for the detection of quantitative trait loci with special consideration of body weight and fat. [In: Ecological genetics in mammals III. G. B. Hartl and J. Markowski, eds]. Acta Theriologica, Suppl. 5: 53-62.

Mouse chromosome 11 was analyzed for the presence of quantitative trait loci (QTL) affecting body weight and fatness. Significant differences in allele frequencies $\left(p<10^{-2}\right)$ between two growth differentiated mouse lines DU6 and DUKs were proven at the marker loci D11Mit120, D11Mit125, and D11Mit126. In families which were derived from crosses between DU6 and DUKs cosegregation of genetic marker alleles and individual trait performance was analyzed. Variance analysis proved highly significant phenotypic differences between genotype groups $\left(p<10^{-5}\right)$, localized in one family in the chromosomal part between D11Mit236 and D11Mit126. These results were confirmed in independent families of parallel crosses. The detected chromosomal region affected both, body weight and fatness.
\end{abstract}

Research Institute for the Biology of Farm Animals, Wilhelm-Stahl-Allee 2, 18196 Dummerstorf, Germany

Key words: quantitative trait loci, growth, fatness, mouse

\section{Introduction}

In animal breeding most traits of interest are of quantitative nature. Their genetic determination comprises the cumulative effect of so called quantitative trait loci (QTL). For many years the efforts of animal breeders have been directed towards an improvement of productive performances. The genetic progresses was backed up by statistical methods of phenotype selection. Despite the dramatic improvements concerning some of the economically important traits in several species of domestic animals several limitations of the traditional selection methods are becoming more and more apparent. Traditional selection in general is hampered in such traits having a low heritability, and in those cases in which several traits are correlated antagonistically.

*Corresponding address: Forschungsinstitut für die Biologie landwirtschaftlicher Nutztiere, FB Molekularbiologie, Wilhelm-Stahl-Allee 2, 18196 Dummerstorf, Germany, fax: 049-38208 68702 , e-mail: brockman@fbn-dummerstorf.de 
Molecular analyses of complex traits may help to solve some of these problems. The availability of complex species specific gene and marker maps provides the capacity to evaluate entire genomes in order to dissect complex traits into their Mendelian entities. Based on genetic markers genome wide scans were applied to informative families for the detection of those genomic loci contributing to complex traits (Lander and Kruglyak 1995). This procedure was used successfully for the detection of single genes contributing to complex traits, eg the obesity gene in mice (Friedman et al. 1991), loci relevant to higher fat storage under high energy nutrition in mice (West et al. 1994a, b) and loci affecting growth on mouse chromosomes 10 and X (Collins et al. 1993, Dragani et al. 1995, Horvat and Medrano 1995).

Our study was focused on the chromosomal localization of QTL affecting the complex traits of body weight and fat content in mice. A major candidate locus in that respect is the growth hormone gene on mouse chromosome 11 . We wanted to examine, if a QTL for body weight and fatness resides on that mouse chromosome, since the growth hormone encoding gene represents a putative candidate gene. Therefore, a mouse line having been selected for high growth (DU6) and a randomly mated control line (DUKs) were analyzed in a two step procedure. Firstly, the line specific allele distributions of genetic markers on mouse chromosome 11 were examined between growth selected (DU6) and unselected control mice (DUKs). Secondly, cosegregation analyses were performed between informative marker alleles and the traits of body weight and abdominal fat content. Families were analyzed, which were derived from crosses between both growth-differentiated mouse line.

\section{Methods}

\section{Animals}

The study was carried out with a randomly mated control (DUKs) and a body weight-selected mouse line (DU6) which has been selected for increased body weight on day 42 of life for 70 generations. Both lines have been generated from a common base population which was originally developed by crossing of four outbred (NMRI orig., Han:NMRI, CFW, CF1) and four inbred populations (CBA/Bln, $\mathrm{AB} / \mathrm{B} \ln , \mathrm{C} 57 \mathrm{BL} / \mathrm{B} \ln , \mathrm{XVII} / \mathrm{Bln})$ in the Research Institute for the Biology of $\mathrm{Farm}$ Animals, Dummerstorf, Germany (Bünger et al. 1983, Schüler 1985). Population sizes were maintained at 80 pairs during selection. Animals were fed ad libitum.

The body weight at 42 days differs in the selected line DU6 from the randomly mated control by 106\% (Bünger et al. 1990). It had progressed far beyond the range of the original outbred population, and it is near to the selection limit. The inbreeding level accounts to $40 \%$. The relative abdominal fat content in line DU6 is twice as high as in the control. Both lines differ in abdominal fat content by more than 3 standard deviations of the unselected control line. The heritabilities for body weight and fat accumulation in these lines were estimated with 0.65 and 0.3 respectively.

For each line, test groups of 20 male mice descending from different litters were analyzed for the detection of specific marker allele distributions between the lines.

To establish second filial (F2) reference families male mice of the body weight-selected line DU6 were crossed with females of the control line DUKs. These families were informative to detect 
Table 1. Design and size of resource family panel. * Two individuals (parental generation) are also included.

\begin{tabular}{llrrrr}
\hline & Family identification & 3 & 4 & 8 & 10 \\
\hline F1 & number of sons & 8 & 4 & 11 & 4 \\
& number of daughters & 9 & 5 & 13 & 5 \\
F2 & 76 & 52 & 189 & 73 \\
\multicolumn{2}{l}{ Total family size* } & 65 & 50 & 152 & 58 \\
& number of males & 160 & 113 & 367 & 142 \\
\hline
\end{tabular}

cosegregation of specific marker genotypes with specific trait performances. Four families were generated, enlarged in sizes by repeated matings of the parents and also of the F1 animals to yield a total of $59 \mathrm{~F} 1$, and $715 \mathrm{~F} 2$ offspring. The individual family designs and sizes are summarized in Table 1. Individuals of each family ie parents, F1, and F2 were genotyped on the marker loci. The body weight of each individual and the abdominal fat pad weight for all F2 were measured on day 42 . Abdominal fat was recorded in males as gonadal fat and as perimetrial fat in females. The ratio of abdominal fat to body mass was defined as relative abdominal fat content.

\section{Microsatellite marker analysis}

DNA was extracted from mouse tail clips using the QIAamp Tissue Kit (Qiagen, Germany). Mouse microsatellite markers (Copeland et al. 1993, Dietrich et al. 1994, Genetic Map of the Mouse 1995) were purchased from Research Genetics (Huntsville, AL) and analyzed by PCR. At microsatellite loci D11Mit120, D11Mit125 and D11Mit126 line specific allele distribution patterns were examined. For the linkage study within families these markers were used and complemented by other markers being located within the detected region: D11Mit236, D11Mit36, D11Mit213, D11Mit180, D11Mit100 and D11Mit214. The parental genotypes had to be checked due to the presence of common alleles in both lines. Depending upon the informativity of parental alleles F2 offspring were genotyped at the corresponding marker locus. Marker positions are given as genetic distance from the centromere in cM units.

The DNA markers were amplified based on a standard PCR protocol as described previously (Das et al. 1996a). Template DNA (20 ng) was amplified in a total reaction volume of $10 \mu \mathrm{l}$. After initial denaturation for $3 \mathrm{~min}$ the DNA was amplified for 25 cycles of $15 \mathrm{sec}$ at $94^{\circ} \mathrm{C}, 1 \mathrm{~min}$ at $55^{\circ} \mathrm{C}$ and 1 min at $70^{\circ} \mathrm{C}$ using Taq polymerase from Appligene (France). PCR fragments were dried, redissolved in formamide loading buffer and separated on $6 \%$ polyacrylamide gels under denaturating conditions. Gels were stained with silver nitrate (Riesner et al. 1989). After initial fixation in a $10 \%$ ethanol / $0.5 \%$ acetic acid solution for $10 \mathrm{~min}$ gels were soaked for $15 \mathrm{~min}$ in a fresh $5.9 \mathrm{mM}$ solution of silver nitrate and developed by an incubation of $10 \mathrm{~min}$ in a solution of $375 \mathrm{mM} \mathrm{NaOH}, 2.3 \mathrm{mM} \mathrm{NaBH} 4$, $0.125 \%$ formaldehyde $(37 \% \mathrm{w} / \mathrm{v})$. The gels were rinsed twice in water and then dried on filter paper (Filtrak Germany).

Marker genotypes were assessed carefully by two independent persons, to avoid false scoring.

\section{Statistical analysis}

Chi-square testing assessed the quantitative differences in the distribution of microsatellite alleles between the lines. For segregation analyses the individual phenotypical values of each family were normalized for the influences of the factors sex and repeated matings. The individual body weight 
performances were sex transformed according to the equation: $x_{i j}^{i}=x_{i j}-\bar{x}_{j}+\bar{x}$, with $x_{i j}^{i}-\operatorname{transformed}$ value for individual $i$ and $\operatorname{sex} j, \bar{x}_{i j}$ - measured body weight for individual $i$ with $\operatorname{sex} j, x_{j}-$ mean body weight within sex $j, \bar{x}-$ mean body weight of both sexes. Analogous transformations corrected for repeated matings. To scrutinize any association between trait associated QTL and the respective microsatellite locus, differences between genotypes were analyzed via variance analysis (ANOVA SAS Institute, 1990). Each family was evaluated separately. Levels of significance for suggestive $(p<2.4$ $\left.\times 10^{-3}\right)$, significant $\left(p<1 \times 10^{-4}\right)$ and highly significant $\left(p<3 \times 10^{-7}\right)$ linkage were used accord ng to Lander and Kruglyak (1995). The calculation of QTL effects based on the estimation of the variance components of the marker genotypes by MIVQUE(0) via SAS.

\section{Results}

\section{Line specific allele distributions}

Initial examinations of our growth and fat differentiated lines DU6 and DUKs showed differences in allele frequencies of microsatellite markers on chromosome 11. At loci D11Mit120, D11Mit125 and D11Mit126 we found line specific allele distributions with significantly different allele frequencies $(p<0.001)$ between both lines (Table 2). The line specific allele distribution is shown at microsatellite loci D11Mit126 and D11Mit120 in Fig. 1. In the selected line DU6 an enrichment of allele 3 of marker D11Mit120, and alleles 1 and 2 of markers D11Mit125 and D11Mit126 were observed. These markers denote two regions on chromosome 11 as being putatively linked with a QTL for body weight. Locus D11Mit120 at 42.4 $\mathrm{cM}$ maps outside of that linkage group comprising the growth hormone gere at $65 \mathrm{cM}$.

Table 2. Allele distribution at marker loci on chromosome 11 between 20 unrelated animals representing the selected (DU6) and unselected (DUKs) mouse lines. ${ }^{\mathrm{a}, \mathrm{b}}$ - significantly different allele distribution at all loci with $p<0.001, \chi^{2}$-test (Rasch et al. 1978).

\begin{tabular}{lcrc}
\hline \multirow{2}{*}{ Locus } & Allele & \multicolumn{2}{c}{ Allele frequency $(\%)$} \\
\cline { 3 - 4 } & & DU6 $^{\text {a }}$ & DUKs $^{\mathrm{b}}$ \\
\hline \multirow{2}{*}{ D11Mit120 } & 1 & 0.0 & 20.0 \\
& 2 & 0.0 & 30.0 \\
D11Mit125 & 3 & 100.0 & 50.0 \\
& 1 & 12.5 & 0.0 \\
& 2 & 50.0 & 17.5 \\
D11Mit126 & 3 & 20.0 & 72.5 \\
& 4 & 17.5 & 10.0 \\
& 1 & 57.5 & 0.0 \\
& 2 & 42.5 & 0.0 \\
& 3 & 0.0 & 72.5 \\
& 4 & 0.0 & 27.5 \\
\hline
\end{tabular}




\section{$\begin{array}{lllllllllllllllllllll}\text { D11Mit126 } & 1 & 2 & 3 & 4 & 5 & 6 & 7 & 8 & 9 & 10 & 11 & 12 & 13 & 14 & 15 & 16 & 17 & 18 & 19 & 20\end{array}$}

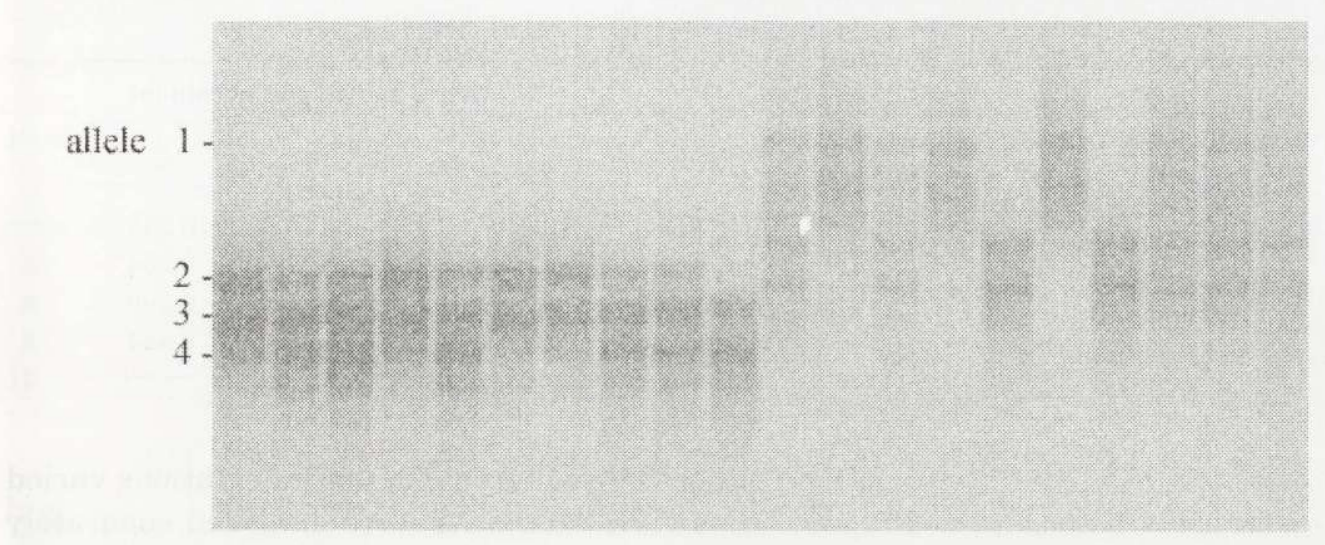

\section{$\begin{array}{lllllllllllllllllllll}\text { D11Mit120 } & 1 & 2 & 3 & 4 & 5 & 6 & 7 & 8 & 9 & 10 & 11 & 12 & 13 & 14 & 15 & 16 & 17 & 18 & 19 & 20\end{array}$}

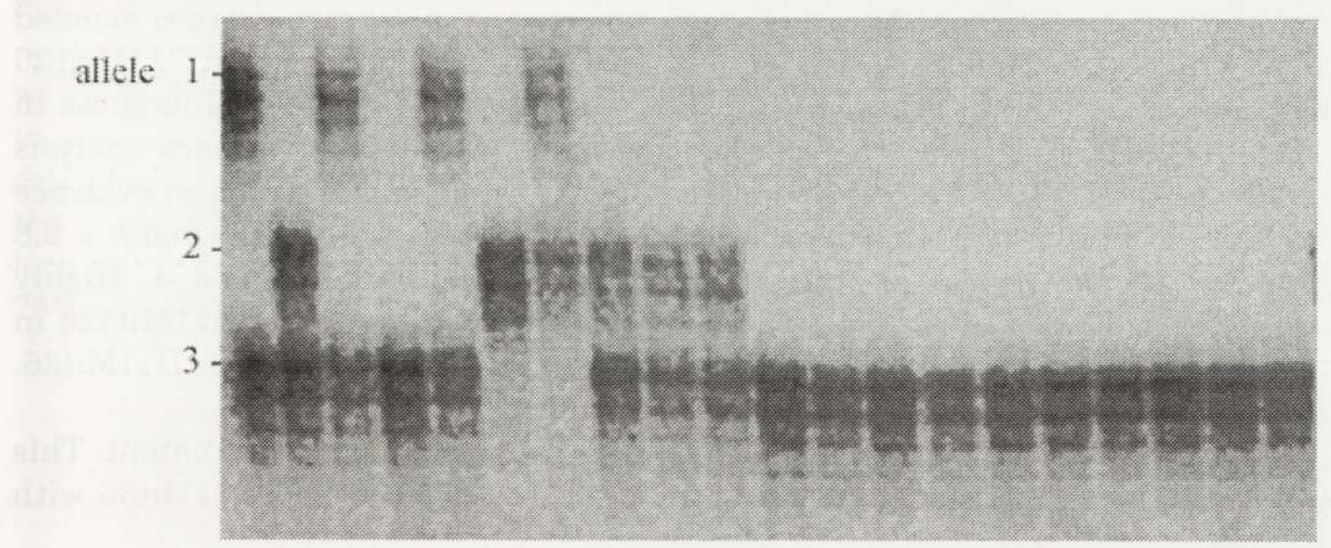

Fig. 1. Line specific allele distribution patterns at microsatellite loci D11Mit126 and D11Mit120 for 10 animals of both lines DUKs (1-10) and DU6 (11-20).

\section{Detection of QTL}

Although selection is intended to represent the main force to generate line specific alleles, genetic drift as a result of small effective populations must not be neglected. Hence, a segregation analysis was carried out within families to exclude non-selection as reason for the line specific allele distribution.

The phenotypic values of the mean body weights and relative abdominal fat contents at 42 days of the F2 populations differed significantly for each of the four families. The mean values ranged between 40.7 and $43.6 \mathrm{~g}$ in body weight and 1.3 
Table 3. Mean $(\bar{x})$ and phenotypic variance $\left(s_{p}^{2}\right)$ of body weight and relative abdominal fat content at 42 days within the second generations of the reference families. The phenotypic data were normalized according to the influences of sex and parity.

\begin{tabular}{rrrrrrrrr}
\hline & \multicolumn{3}{c}{ Body weight } & & & \multicolumn{3}{c}{ Relative abdominal fat content } \\
\cline { 2 - 3 } \cline { 7 - 8 } Family & $n$ & $\bar{x}(\mathrm{~g})$ & $s_{p}^{2}$ & & \multicolumn{1}{c}{$n$} & $\bar{x}(\mathrm{~g})$ & $s_{p}^{2}$ \\
\hline 3 & 141 & 40.73 & 20.34 & & 138 & 1.416 & 0.245 \\
4 & 102 & 40.69 & 15.70 & & 92 & 1.293 & 0.254 \\
8 & 341 & 41.00 & 22.83 & & 338 & 1.826 & 0.399 \\
10 & 131 & 43.60 & 24.91 & & 131 & 2.019 & 0.484 \\
\hline
\end{tabular}

to $2.0 \%$ in relative abdominal fat content. The values of standard deviations varied up to $100 \%$ between the F2 populations. Thus families were analyzed separately for linked inheritance of marker genotype and trait performance. The phenotypic data of all families are given in Table 3.

The data were analyzed for the presence of detectable QTL influencing body weight and relative abdominal fat content using variance analyses. The statistical tests on linkage between individual body weights and marker genotypes denoted a large region on chromosome 11 between the markers D11Mit236 and D11Mit126 affecting body weight performances. The levels of significance for differences in body weight between groups of marker genotypes obtained by variance analysis (ANOVA) are given in Table 4. Marker D11Mit36 provided the strongest evidence for linkage with body weight in families 8 and 10 at $p<2.2 \times 10^{-13}$ and $p<2.8$ $\times 10^{-7}$ respectively. The results were confirmed in families 3 and 4 . Highly significant linkage to body mass was also evident at marker locus D11Mit126 in family 8 at $p<2.2 \times 10^{-8}$ located at a distance of $20 \mathrm{cM}$ away from D11Mit36. Significant linkage at this locus was proven in family $10\left(p<10^{-6}\right)$.

The detected region showed also effect on relative abdominal fat content. This QTL is being located in the region between loci D11Mit236 and D11Mit36 with

Table 4. Significance limits of the $F$-test (ANOVA) for differences in body weight performances between genotype groups of all markers tested. n.i. - parental genotypes are not informative, ns - not significant.

\begin{tabular}{clllllllll}
\hline & \multicolumn{8}{c}{ Microsatellite D11 } \\
\cline { 2 - 10 } Family & Mit236 & Mit36 & Mit120 & Mit213 & Mit125 & Mit126 & Mit180 & Mit100 & Mit214 \\
& $17.9 \mathrm{cM}$ & $42.4 \mathrm{cM}$ & $42.4 \mathrm{cM}$ & $52.6 \mathrm{cM}$ & $62.6 \mathrm{cM}$ & $63.7 \mathrm{cM}$ & $69.5 \mathrm{cM}$ & $70.6 \mathrm{cM}$ & $77.3 \mathrm{cM}$ \\
\hline 3 & ns & $9.9 \times 10^{-5}$ & n.i. & n.i. & 0.039 & ns & ns & n.i. & n.i. \\
4 & 0.0158 & 0.0040 & 0.0074 & n.i. & 0.0018 & 0.0020 & 0.0005 & n.i. & ns \\
8 & $4.5 \times 10^{-9}$ & $2.2 \times 10^{-13}$ & $1.4 \times 10^{-12}$ & n.i. & $3.3 \times 10^{-10}$ & $2.2 \times 10^{-8}$ & n.i. & n.i. & n.i. \\
10 & $2.3 \times 10^{-5}$ & $2.8 \times 10^{-7}$ & n.i. & $1.2 \times 10^{-5}$ & $7.5 \times 10^{-6}$ & $1 \times 10^{-6}$ & 0.013 & n.i. & n.i. \\
\hline
\end{tabular}


Table 5. Significance limits of the $F$-test (ANOVA) for differences in relative abdominal fat content between the genotype groups of all markers tested. n.i. - parental genotypes are not informative, ns - not significant.

\begin{tabular}{llllllllll}
\hline & \multicolumn{8}{c}{ Microsatellite D11 } \\
\cline { 2 - 10 } Family & Mit236 & Mit36 & Mit120 & Mit213 & Mit125 & Mit126 & Mit180 & Mit100 & Mit214 \\
& $17.9 \mathrm{cM}$ & $42.4 \mathrm{cM}$ & $42.4 \mathrm{cM}$ & $52.6 \mathrm{cM}$ & $62.6 \mathrm{cM}$ & $63.7 \mathrm{cM}$ & $69.5 \mathrm{cM}$ & $70.6 \mathrm{cM}$ & $77.3 \mathrm{cM}$ \\
\hline 3 & 0.0076 & 0.0267 & n.i. & n.i. & ns & ns & ns & n.i. & n.i. \\
4 & 0.0004 & ns & ns & n.i. & ns & ns & 0.0417 & n.i. & ns \\
8 & ns & ns & ns & n.i. & ns & ns & n.i. & n.i. & n.i. \\
10 & 0.0211 & 0.0008 & n.i. & 0.0211 & 0.0069 & 0.0025 & ns & n.i. & n.i. \\
\hline
\end{tabular}

high statistical probability (Table 5). In family 4 suggestive linkage for a QTL affecting abdominal fat content was proven at marker locus D11Mit236 $(p<$ $0.0004)$, in family 10 at locus D11Mit36 ( $p<0.0008)$.

In each case the paternal marker genotype(s) descending from the growth selected line was associated with the highest mean body weight value and highest mean abdominal fat content value in the F2 generation. The maternal genotypes - derived from the control - showed association to the group with the least body weight respectively. The effect of locus D11Mit36 on body weight accounts for 11 to $27 \%$ of the variance within the F2 offspring (Table 6). The highest value was estimated in family 10. In the same family this marker locus contributes $12 \%$ to the variation of relative abdominal fat content. The estimated effect of marker locus D11Mit236 on abdominal fat content was highest in family 4, amounting to $25 \%$.

Table 6. Effect of marker linked QTL on body weight and abdominal fat content as ratio of estimated variance compound between the genotype groups to total variance within F2 generations. n.d. - not determined.

\begin{tabular}{lrcc}
\hline Marker & Family & Body weight $(\%)$ & Abdominal fat content (\%) \\
\hline \multirow{2}{*}{ D11Mit236 } & 3 & n.d. & 8 \\
& 4 & n.d. & 25 \\
& 8 & n.d. & 0 \\
D11Mit36 & 10 & n.d. & 5 \\
& 3 & 10.6 & 0 \\
& 4 & 16.7 & 0 \\
D11Mit126 & 8 & 15.2 & 0 \\
& 10 & 27.1 & 11.8 \\
& 3 & 0 & 0 \\
& 4 & 10.5 & 0 \\
& 8 & 10.0 & 15.0 \\
\hline
\end{tabular}




\section{Discussion}

With the hypothesis, that selection causes a linkage disequilibrium between specific marker loci and QTL controlling body weight and fat accumulation, selection is expected to generate line specific distribution of microsatellite alleles. Initial examinations of microsatellite markers in the two mouse lines DU6 and DUKs indicated that long term trait-selection in mice leads to remarkable differences in allele frequencies at single microsatellite loci between lines. That corresponds with earlier investigations based on DNA fingerprinting. In those experiments line specific fingerprint patterns showed 2-6 line specific bands depending on the combination of restriction enzymes and probes (Brockmann et al. 1993, 1996).

However, in these examinations the line specific distribution of alleles at the microsatellite loci on chromosome 11 were only an initial hint on a QTL for body weight and fatness, putatively being localized on this chromosome.

Yet, the relatively high number of microsatellite loci with line specific alleles on chromosome 11 might reflect influences of genetic drift on the development of both, the control and selected lines. Although Keightley et al. $(1993,1996)$ detected on this chromosome QTL as judged from frequency changes of marker alleles in selected mouse lines, a linkage analysis within families is necessary for precise QTL mapping.

In our family study, QTL for body weight and relative abdominal fat content were identified on mouse chromosome 11. The detected QTL on chromosome 11 showed a high effect on growth, indicating a major role of this region for growth. Although previously the Gh-gene was assumed to be the main putative candidate gene in the detected distal chromosomal region, neither RFLP (restriction fragment length polymorphism) nor SSCP (simple sequence conformation polymorphism) analyses between individuals of both lines contributing to our crosses showed any polymorphism in the gene or promoter structure (Das et al. 1996b). These results of the gene analysis suggested that the growth hormone gene locus itself is probably not the identified QTL. Conceivably, either a regulatory sequence of the multicomponent control region (Jones et al. 1995) not having been detected in our assay, or even other genes cause the observed growth differences.

Furthermore, our study suggests that a locus affecting relative abdominal fat content is located in the proximal region of chromosome 11. Until now, no candidate gene possibly causing this effect has been identified in this region.

For the identification of QTL we obtained different results in single families generated by crossing a selected with a randomly mated line. These differences reflect heterogeneity of the genomes between the individuals contributing to each line. Family specific results are mainly due to the heterogeneity in the control line. Selection maximizes the fixation of alleles contributing to high selection response. However, residual heterozygosity in the selected line might be maintained 
due to the combined realization of the high performance QTL allele and alleles contributing to other beneficial traits, eg reproduction.

Using the advantage of known location of markers linked to QTL in the mouse genome it is now possible to transfer some of the results obtained in mice to farm animal research, exploiting comparative maps of mouse-human-pig and cattle.

Acknowledgements: Excellent technical assistance was provided by Hannelore Tychsen for help in DNA preparation and genotyping. This work was supported by the Deutsche Forschungsgemeinschaft, Grant No BR 1285/1-2

\section{References}

Brockmann G., Buitkamp J., Bünger L., Epplen J. T. and Schwerin M. 1993. DNA Fingerprinting of trait-selected mouse lines and linkage analysis in reference families. [In: DNA fingerprinting: approaches and applications, Chakraborty. J. T. Epplen, A. J. Jeffreys and S. D. J. Pena, eds]. Birkhäuser Verlag, Basel: 403-410.

Brockmann G., Buitkamp J., Teuscher F., Bünger L., Epplen J. T. and Schwerin M. 1996. Multilocus fingerprint bands are associated with quantitative traits in selected mouse lines: linkage analysis in reference families. Archiv für Tierzucht 39: 477-487.

Bünger L., Herrendörfer G. and Renne U. 1990. Results of long term-selection for growth traits in laboratory mice. Proceedings of the 4th World Congress on Genetics of Applied Livestock Prodction, Edinburgh, XIII: 321-324.

Bünger L., Schüler L., Kupatz B. and Renne U. 1983. Selection for growth in laboratory mice. II. Dorect selection response. Archiv für Tierzucht 26: 281-293.

Collins A. C., Martin I. C. A. and Kirkpatrik B. W. 1993. Growth quantitative trait loci (QTL) on mouse chromosome 10 in a Quackenbrush- Swiss $\times$ C57BL/6J backcross. Mammalian Genome 4: $454-458$.

Copeland N.G., Gilbert D. J., Jenkins N. A., Nadeau J. T., Eppig L. J., Maltais J. C., Miller W. F., Dietrich W. F., Steen R. G., Lincoln S. E., Weaver A., Joyce D. C., Merchant M., Wessel M., Katz H., Stein L. D., Reeve M. P., Daly M. J., Dredge R. D., Marquis A., Goodman N. and Lander E. S. 1993. Genome maps IV. Science 262: 67.

Das P., Brockmann G., Meyer L., Renne U., Freyer G. and Schwerin M. 1996a. The effect of the distal chromosome 11 on body weight in mice under special consideration of the growth hormone gene locus. Archiv für Tierzucht 39: 185-194.

Das P., Meyer L., Seyfert M., Brockmann G. and Schwerin M. 1996b. Structure of growth hormone-encoding gene and its promoter in mice. Gene 169: 209-213.

Dragani T. A., Zeng Z. B., Canzian F., Gariboldi M., Ghilarducci M. T., Mananti G. and Pierotti M. A. 1995. Mapping of body weight loci on mouse chromosome X. Mammalian Genome 6: 11 .

Dietrich W. F., Miller J. C., Steen R. G., Merchand M., Damron D., Nahf R., Yoyce D. C., Wessel M., Dredge R. D. 1994. A genetic map of the mouse with 4006 simple sequence length polymorphisms. Nature Genetics 7: 220-245.

Friedman J. M., Leibel R. L., Siegel D. S., Walsh J. and Bahary N. 1991. Molecular mapping of the mouse $o b$ mutation. Genomics 11: 1054-1062.

Genetic Map of the Mouse 1995. Whitehead Institute/MIT Center for genome research, database release April.

Horvat S. and Medrano J. F. 1995. Intervall Mapping of high growth (hg), a major locus that increases weight gain in mice. Genetics 139: 1737-1748.

Jones B. K., Monks B. R., Liebhaber S. A. and Cooke N. E. 1995. The human growth hormone gene is regulated by a multicomponent locus control region. Molecular Cell Biology 15: 7010-7021.

Keightley P. D. and Bulfield G. 1993. Detection of quantitative trait loci from frequency changes of marker alleles under selection. Genetical Research 62: 195-203. 
Keightley P. D., Hardge T., May L. and Bulfield G. 1996 A genetic map of quantitative trait loci for body weight in the mouse. Genetics 142: 227-235.

Lander E. S. and Kruglyak L. 1995. Genetic dissection of complex traits: guidelines for interpreting and reporting linkage results. Nature Genetics 11: 241-247.

Rasch D., Herrendörfer G., Bock J. and Busch, K. 1978. Verfahrensbibliothek Versuchsplanung und -auswertung, Band 2. Deutscher Landwirtschaftsverlag Berlin: 884.

Riesner D., Steger G., Zimmat R., Owens R. A., Wagenhöfer M., Hillen W., Vollbach S. and Henco K. 1989. Temperature-gradient gel electrophoresis of nucleic acids. Analysis of conformational transitions, sequence variations, and protein-nucleic acid interactions. Electrophoresis 10: 377-389.

SAS, 1990. SAS user's guide. statistics. SAS Instruments Incorporation, Cary, NC.

Schüler L. 1985. Mouse strain Fzt.:Du and its use as model in animal breeding research. Archiv für Tierzucht 28: $357-363$.

West D. B., Goudey-Lefevre J., York B. and Truett G. E. 1994a. Dietary obesity linked to genetic loci on chromosome 9 and 15 in a polygenic mouse model. Journal of Clinical Investigation 94 $1410-1416$

West D. B., Waguespack J., York B., Goudey-Lefevre J. and Price R. A. 1994b. Genetics of dietary obesity in AKR/J $\times \mathrm{SWR} / J$ mice, segregation of the trait and identification of a linked locus on chromosome 4. Mammalian Genome 5: 546-552.

Received 10 June 1998, accepted 15 July 1998. 